Centre for Decision Research and Experimental Economics

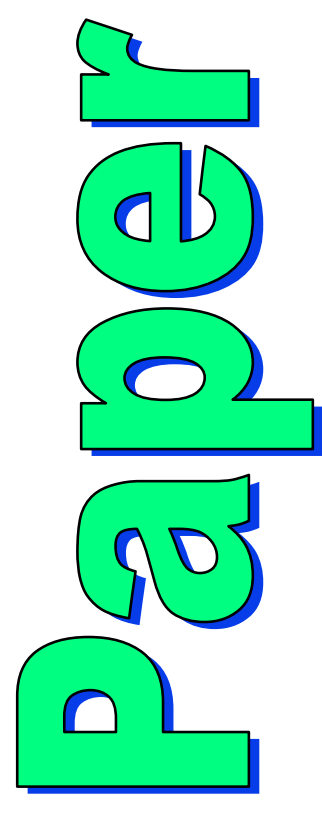

CeDEx Discussion Paper No. 2007-12

Discussion Paper Series

ISSN 1749-3293

The Paradox of New Members in the Council of Ministers: A Noncooperative Approach

Maria Montero

December 2007 
The focus for the Centre is research into individual and strategic decision-making using a combination of theoretical and experimental methods. On the theory side, members of the Centre investigate individual choice under uncertainty, cooperative and non-cooperative game theory, as well as theories of psychology, bounded rationality and evolutionary game theory. Members of the Centre have applied experimental methods in the fields of Public Economics, Individual Choice under Risk and Uncertainty, Strategic Interaction, and the performance of auctions, markets and other economic institutions. Much of the Centre's research involves collaborative projects with researchers from other departments in the UK and overseas.

Please visit http://www.nottingham.ac.uk/economics/cedex/ for more information about the Centre or contact

Karina Whitehead

Centre for Decision Research and Experimental Economics

School of Economics

University of Nottingham

University Park

Nottingham

NG7 2RD

Tel: +44 (0) 1159515620

Fax: +44 (0) 1159514159

karina.whitehead@nottingham.ac.uk

The full list of CeDEx Discussion Papers is available at

http://www.nottingham.ac.uk/economics/cedex/papers/index.html 


\title{
The Paradox of New Members in the Council of Ministers: A Noncooperative Approach
}

\author{
Maria Montero*
}

December 2007

\begin{abstract}
Power indices suggest that adding new members to a voting body may increase the power of an existing member, even if the number of votes of all existing members and the decision rule remain constant. This phenomenon is known as the paradox of new members. This paper shows that the paradox has theoretically occurred in the EU using the leading model of legislative bargaining. Furthermore, it is possible for a majority of members to be in favor of enlargement, even if voters are bargaining over a fixed budget.
\end{abstract}

Keywords: legislative bargaining, weighted voting, power measures, EU enlargement, paradox of new members.

J.E.L. Classification Numbers: C71, C72, C78.

${ }^{*}$ University of Nottingham, School of Economics, University Park, Nottingham NG7 2RD (United Kingdom); maria.montero@nottingham.ac.uk. I'm grateful to Alex Possajennikov and Martin Sefton for helpful comments. 


\section{Introduction}

The paradox of new members was identified by Brams and Affuso (1976) and refers to the possibility that adding new members to a voting body may increase the power of an existing member, even if the number of votes of all existing members and the decision rule remain constant. Brams and Affuso based their analysis on the application of Shapley and Banzhaf power indices to weighted voting games. In later papers (Brams and Affuso, 1985a, 1985b) they showed that the paradox has theoretically occurred in the EEC (now EU) Council of Ministers.

Drouvelis, Montero and Sefton (2007) show that the paradox can occur as an equilibrium of the Baron-Ferejohn (1989) model of legislative bargaining. In this model, the voters bargain over the division of a fixed budget by making and voting on proposals, and a voter's power can be measured by its expected equilibrium payoff. ${ }^{1}$

This paper takes a noncooperative approach to voting in the Council of Ministers using the same model. The equilibrium of the bargaining game is analyzed for the Council of Ministers in 1958, 1973 and 1981. Comparing the countries' expected payoffs before and after each enlargement, it is observed that at least one existing member is better-off in each of the two enlargements. Furthermore, the countries that gain with the 1981 enlargement had a majority in the 1973 Council. Thus, if qualified majority voting had been used to decide on enlargement, the new member would have been admitted even if the countries were bargaining over a fixed pie.

\section{The noncooperative bargaining procedure}

There is a budget of size 1 to be divided by majority rule between $n$ players. Player $i$ has $w_{i}$ votes and $q$ votes are needed to achieve a majority. We will denote a weighted majority game by $\left[q ; w_{1}, \ldots, w_{n}\right]$. A group of players $S$ with $\sum_{i \in S} w_{i} \geq q$ is called a winning coalition; a winning coalition such that $\sum_{j \in S \backslash\{i\}} w_{j}<q$ for all $i$ is called a minimal winning coalition. A player

\footnotetext{
${ }^{1}$ This concept of power is sometimes labelled P-power (Felsenthal and Machover, 1998).
} 
that does not belong to any minimal winning coalition is a dummy player. A player that belongs to all minimal winning coalitions is a veto player.

Bargaining proceeds as follows: At every round $t=1,2, \ldots$, Nature randomly selects a proposer (each player is selected with probability $\frac{1}{n}$ ). This player proposes a distribution of the budget $\left(x_{1}, \ldots, x_{n}\right)$ with $x_{i} \geq 0$ for all $i$ and $\sum_{i \in N} x_{i}=1$. The proposal is voted upon immediately (closed rule). If the sum of votes in favor of the proposal is at least $q$, the proposal is implemented and the game ends; otherwise the game proceeds to the next period in which Nature selects a new proposer (again each player is selected with probability $\frac{1}{n}$ ). Players are risk-neutral and do not discount future payoffs.

A (pure) strategy for player $i$ is a sequence $\sigma_{i}=\left(\sigma_{i}^{t}\right)_{t=1}^{\infty}$, where $\sigma_{i}^{t}$, the $t$ th round strategy of player $i$, prescribes

1. A proposal $x$.

2. A response function assigning "yes" or "no" to all possible proposals by the other players.

The solution concept is stationary subgame perfect equilibrium (SSPE). Stationarity requires that players follow the same strategy at every round $t$ regardless of past offers and responses to past offers. Banks and Duggan (2000) show that an SSPE always exists in this type of bargaining model. Eraslan and McLennan (2006) show that all SSPE lead to the same expected equilibrium payoffs.

In the absence of veto players, expected payoffs must be positive for all player types (see e.g. Lemma 1.4 of Appendix A in Drouvelis, Montero and Sefton (2007)). None of the voting games we are going to analyze has veto players.

\subsection{Three voting bodies}

Table 1, adapted from Felsenthal and Machover (2001), shows the weighted majority voting games associated to the original European Community in 1958, 1973 and 1981. 


\begin{tabular}{|l|l|l|l|}
\hline Country & $\mathbf{1 9 5 8}$ & $\mathbf{1 9 7 3}$ & $\mathbf{1 9 8 1}$ \\
\hline Germany & 4 & 10 & 10 \\
Italy & 4 & 10 & 10 \\
France & 4 & 10 & 10 \\
Netherlands & 2 & 5 & 5 \\
Belgium & 2 & 5 & 5 \\
Luxemburg & 1 & 2 & 2 \\
UK & - & 10 & 10 \\
Denmark & - & 3 & 3 \\
Ireland & - & 3 & 3 \\
Greece & - & - & 5 \\
Quota & 12 & 41 & 45 \\
Total votes & 17 & 58 & 63 \\
Quota (\%) & 70.59 & 70.69 & 71.43 \\
\hline
\end{tabular}

Table 1: Weights and quota in the Council of Ministers

We now calculate the equilibrium payoffs of the bargaining game for each of these voting bodies. Since equilibrium payoffs are unique, it will suffice to find one equilibrium strategy combination (all other equilibrium combinations lead to the same payoffs). From now on we restrict ourselves to symmetric strategies: all players of the same type follow the same strategy and are treated symmetrically by other players' strategies.

In a stationary equilibrium, a player's expected payoff given that a proposal is rejected (the continuation value) equals his expected equilibrium payoff at the beginning of the game. It is optimal for each player to accept any offer that gives him at least his continuation value as a responder. As a proposer, player $i$ looks for the cheapest group of players controlling at least $q-w_{i}$ votes, and makes a proposal allocating to these players their continuation values and keeping the remainder for himself. Following common practice, we will refer to the proposer together with the players that are offered their continuation values as the "proposed coalition", and, if the proposal is passed, as the "coalition that forms". 
Two conditions must be satisfied in equilibrium: strategies must be optimal given expected payoffs, and expected payoffs must be consistent with the strategies. To find the equilibrium expected payoffs, we will make hypotheses about them (e.g., the expected payoff of a player with 4 votes is twice the expected payoff of a player with 2 votes) and then construct strategies that are optimal given the hypotheses and that lead to payoffs satisfying the hypotheses.

\subsection{Equilibrium of game $[12 ; 4,4,4,2,2,1]$}

There are four minimal winning coalitions in this game: one coalition of type [444] and three coalitions of type [4422]. The player with 1 vote does not belong to any minimal winning coalition.

Denote expected equilibrium payoffs by $x$ (for a player with 4 votes), $y$ (for a player with 2 votes) and $w$ (for the player with 1 vote).

Suppose equilibrium payoffs are such that $x=2 y$. Under this hypothesis, a player with 4 votes is indifferent between paying $2 x$ and forming a coalition of type [444] and paying $x+2 y$ and forming one of the two coalitions of type [4422] to which he belongs. Denote by $\lambda$ the probability that a given player with 4 votes proposes [444] (conditional on being proposer). The probability of proposing each of the two coalitions of type [4422] is then $\frac{1-\lambda}{2}$. A player with 2 votes needs to buy 10 votes, and the best way to do this is to form a coalition of type [4422] (a coalition of type [4442] would be too expensive under the hypothesis $x=2 y$ ). There are three such coalitions, each proposed with probability $\frac{1}{3}$. The player with 1 vote needs to buy 11 votes, and is indifferent between forming coalition [4441] and forming a coalition of type [44221]. Denote the probability of proposing [4441] by $\mu$; then each of the three [44221] coalitions is proposed with probability $\frac{1-\mu}{3}$. Table 2 shows the probability that each player type proposes each of the coalition types, with the number of available coalitions for that player type in parentheses. Because the proposer must be included in the coalition, the number of available coalitions of each type may depend on the proposer's 
type.

$$
\begin{array}{clllll} 
& \multicolumn{5}{c}{\text { Coalition type }} \\
& {[4]} & \lambda(1) & \frac{1-\lambda}{2}(2) & - & - \\
\text { Player type } & {[2]} & - & \frac{1}{3}(3) & - & - \\
& {[1]} & - & - & \mu(1) & \frac{1-\mu}{3}(3)
\end{array}
$$

Expected equilibrium payoffs are determined by these strategies. Consider a player with 4 votes. With probability $\frac{1}{6}$ he is selected to be proposer and obtains a payoff of $1-2 x$ (this is the proposer's payoff regardless of whether he proposes [444] or [4422] because $x=2 y$ ). With probability $\frac{2}{6}$, one of the two other player with 4 votes is selected, and the player receives a proposal with probability $\lambda+\frac{1-\lambda}{2}$. With probability $\frac{2}{6}$ one of the two players with 2 votes is selected and proposes each coalition of type [4422] with probability $\frac{1}{3}$. A given player with 4 votes belongs to two of these three coalitions, and thus receives a proposal with probability $\frac{2}{3}$. With probability $\frac{1}{6}$ the player with 1 vote is selected and proposes to the player with 4 votes with probability $\mu+\frac{2}{3}(1-\mu)$. The equations for $y$ and $w$ can be derived analogously. Together with the postulated condition $x=2 y$, we have the following system of equations

$$
\begin{aligned}
x & =\frac{1}{6}(1-2 x)+\frac{2}{6}\left(\lambda+\frac{1-\lambda}{2}\right) x+\frac{2}{6} \frac{2}{3} x+\frac{1}{6}\left(\mu+\frac{2}{3}(1-\mu)\right) x \\
y & =\frac{1}{6}(1-2 x-y)+\frac{3}{6}(1-\lambda) y+\frac{1}{6} y+\frac{1}{6}(1-\mu) y \\
w & =\frac{1}{6}(1-3 x) \\
x & =2 y
\end{aligned}
$$

The solution to this system of equations is $0 \leq \mu \leq 1, \lambda=\frac{12-5 \mu}{15}, x=\frac{10}{42}$, $y=\frac{5}{42}, w=\frac{2}{42}$. Notice that even though Luxemburg is a dummy player its expected equilibrium payoff is positive because it is allowed to make proposals. 


\subsection{Equilibrium of game $[41 ; 10,10,10,10,5,5,3,3,2]$}

The 1973 enlargement changed the voting game from $[12 ; 4,4,4,2,2,1]$ to $[41 ; 10,10,10,10,5,5,3,3,2]$. Three new members were added and the weights of all pre-existing members were multiplied by 2.5 , with the exception of the smallest member (Luxemburg), whose votes were multiplied by 2 . The percentage of the total votes required to pass a proposal remained essentially constant (keeping it exactly constant would lead to a quota of 40.94, which has the same implications as a quota of 41). If Luxemburg's votes had been multiplied by 2.5 , any incumbent being better-off would be an instance of the paradox of new members. The fact that Luxemburg's votes were multiplied by only 2 seems to make it more difficult for Luxemburg to be better-off after the enlargement. ${ }^{2}$ However, we will see that Luxemburg's expected equilibrium payoff increases after the enlargement in the Baron-Ferejohn model.

There are 25 minimal winning coalitions of six possible types: [10 1010

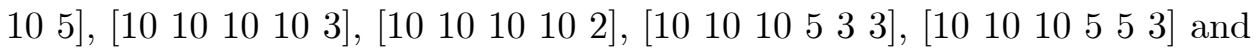
$\left[\begin{array}{llllll}10 & 10 & 10 & 5 & 5 & 2\end{array}\right]$.

Expected equilibrium payoffs will be denoted by $x$ (players with 10 votes), $y$ (players with 5 votes), $z$ (players with 3 votes) and $w$ (player with 2 votes). Postulate an equilibrium with $x>y>z>w, x=2 y$ and $y+w=2 z$. Then the following types of minimal winning coalitions are the cheapest: [10 10101010 2], [10 10101055 2], [10 $\left.1010 \begin{array}{llll}10 & 5 & 3 & 3\end{array}\right]$. Other minimal winning coalition types are too expensive to form.

\footnotetext{
${ }^{2}$ In fact, $[41 ; 10,10,10,10,5,5,3,3,2]$ and $[41 ; 10,10,10,10,5,5,3,3,1]$ have the same winning coalitions, thus Luxemburg's votes might as well have remained constant.
} 
Equilibrium strategies are summarized by the following table

\section{Coalition type}

$\left[\begin{array}{lllll}10 & 10 & 10 & 10 & 2\end{array}\right] \quad\left[\begin{array}{llllll}10 & 10 & 10 & 5 & 5 & 2\end{array}\right] \quad\left[\begin{array}{llllll}10 & 10 & 10 & 5 & 3 & 3\end{array}\right]$

$\begin{array}{rllll} & {[10]} & \lambda(1) & \mu(3) & \frac{1-\lambda-3 \mu}{6}(6) \\ \text { Player type } & {[5]} & - & \theta(4) & \frac{1-4 \theta}{4}(4) \\ & {[3]} & - & - & \frac{1}{8}(8) \\ {[2]} & \rho(1) & \frac{1-\rho}{4}(4) & -\end{array}$

The four equations for expected payoffs together with the two conditions we have postulated form the following system of equations:

$$
\begin{aligned}
x & =\frac{1}{9}(1-3 x-w)+\frac{3}{9}\left(\lambda+\frac{2}{3}(1-\lambda)\right) x+\frac{4}{9} \frac{3}{4} x+\frac{1}{9}\left(\rho+\frac{3}{4}(1-\rho)\right) x \\
y & =\frac{1}{9}(1-3 x-2 z)+\frac{4}{9}\left(3 \mu+\frac{1-\lambda-3 \mu}{2}\right) y+\frac{1}{9} 4 \theta y+\frac{2}{9} \frac{1}{2} y+\frac{1}{9}(1-\rho) y \\
z & =\frac{1}{9}(1-3 x-y-z)+\frac{4}{9}(1-\lambda-3 \mu) z+\frac{2}{9}(1-4 \theta) z+\frac{1}{9} z \\
w & =\frac{1}{9}(1-4 x)+\frac{4}{9}(\lambda+3 \mu) w+\frac{2}{9} 4 \theta w \\
x & =2 y \\
2 z & =y+w
\end{aligned}
$$

Again there are infinitely many solutions for the equilibrium strategies, but a unique solution for $x, y, z$ and $w$. The (unique) equilibrium expected payoffs are $x=\frac{67-\sqrt{73}}{368} \approx 0.159, y=\frac{67-\sqrt{73}}{736} \approx 0.079, z=\frac{9 \sqrt{73}+133}{2944} \approx 0.071$, $w=\frac{11 \sqrt{73}-1}{1472} \approx 0.063$. There are many possible values for the strategies. Setting $\mu=0$ and $\theta=\frac{1}{4}$ we obtain $\lambda=\frac{11-\sqrt{73}}{8} \approx 0.31$ and $\rho=\frac{\sqrt{73}-8}{3} \approx 0.18$.

Luxemburg has stopped being a dummy player, and this increases its equilibrium payoffs.

Perhaps surprisingly, expected payoffs for countries with 2, 3 and 5 votes do not differ much. Intuition dictates that a country with 5 votes and a combination of two countries with 3 and 2 votes respectively are interchangeable and ought to have the same expected payoff. However, there are not enough 
players for this to be feasible. There is no minimal winning coalition in which a player with 3 votes and a player with 2 votes appear together. ${ }^{3}$ Minimal winning coalitions including a player with 5 votes already include the player with 2 votes (coalitions of type [10 101010552 ) ) or both of the players with 3 votes (coalitions of type [10 101010533 ]) or are too expensive to be relevant (in [ [ $\left.\begin{array}{lllll}10 & 10 & 10 & 10 & 5\end{array}\right]$ and [ [ $\left.\begin{array}{llllll}10 & 10 & 10 & 5 & 5 & 3\end{array}\right]$ a player with 5 votes could be replaced by a combination of two players, but then the player with 2 votes would be superfluous).

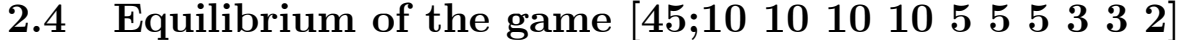

In 1981 Greece entered the European Community with 5 votes and the quota was raised to 45 . The voting weights of all other countries were left unchanged, and the percentage of votes required to achieve a majority was essentially unchanged since $\frac{41}{58} \times 63 \approx 44.53$.

This new game is radically different to the previous one and easier to analyze. First, a player with 3 votes and a player with 2 votes have become interchangeable. The new voting game is equivalent to the game $[18 ; 4,4,4,4,2,2,2,1,1,1]$. Second, the possibility of replacing a player with 5 votes by a combination of players with 3 and 2 votes (or 3 and 3 votes) has become relevant.

As before, we denote expected payoffs by $x, y$ and $z$. Since players with 3 votes and players with 2 votes have become interchangeable, they both have the same expected payoff $z$. To simplify the search for equilibrium, we limit ourselves to strategies in which the players with 3 votes and the player with 2 votes follow the same strategy and are treated symmetrically by other players.

If we postulate $x=2 y$ and $y=2 z$, this means that expected payoffs are determined by these equations together with $4 x+3 y+3 z=1$. These values are $x=0.16, y=0.08, z=0.04$. All we need is to verify that there are equilibrium strategies supporting those payoffs.

\footnotetext{
${ }^{3}$ This can only happen if the weighted majority game is not strong (i.e., we can divide the players in two groups in such a way that both groups have less than $q$ votes). In strong games, any two players share membership of at least one minimal winning coalition.
} 
Under the hypotheses $x=2 y$ and $y=2 z$, all minimal winning coalitions are equally cheap. There are 46 minimal winning coalitions ${ }^{4}$ of 6 possible types ( 4 types if we take into account that players with 2 and 3 votes are interchangeable): [10 10101010 5], [10 $\left.10 \begin{array}{lllll}10 & 10 & 10 & 3\end{array}\right]$, [10 $\left.10 \begin{array}{lllll}10 & 10 & 3\end{array}\right]$, [10

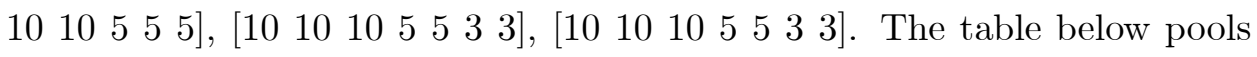
players with 2 and 3 votes.

\begin{tabular}{|c|c|c|c|c|}
\hline & {$\left[\begin{array}{lllll}10 & 10 & 10 & 10 & 5\end{array}\right]$} & $\begin{array}{l}{\left[\begin{array}{llllll}10 & 10 & 10 & 10 & 3 & 3\end{array}\right]} \\
{\left[\begin{array}{llllll}10 & 10 & 10 & 10 & 3 & 2\end{array}\right]}\end{array}$ & {$\left[\begin{array}{llllll}10 & 10 & 10 & 5 & 5 & 5\end{array}\right]$} & $\begin{array}{l}{\left[\begin{array}{lllllll}10 & 10 & 10 & 5 & 5 & 3 & 3\end{array}\right]} \\
{\left[\begin{array}{llllll}10 & 10 & 10 & 5 & 5 & 3\end{array}\right]}\end{array}$ \\
\hline [10] & $\lambda(3)$ & $\mu(3)$ & $\theta(3)$ & $1-3 \lambda-3 \mu-3$ \\
\hline$[10]$ & $\lambda(J)$ & $\mu(J)$ & $\theta(3)$ & $\begin{array}{l}27 \\
1-\rho-4 \sigma\end{array}$ \\
\hline$[3 / 2]$ & $\begin{array}{l}P(1) \\
-\end{array}$ & $\tau(2)$ & - & $\frac{1-24}{24}(24)$ \\
\hline
\end{tabular}

We can simplify the search further by looking for equilibria with $\mu=$ $\theta=\rho=\tau=0$. The strategy table becomes

\begin{tabular}{|c|c|c|c|c|}
\hline & {$\left[\begin{array}{lllll}10 & 10 & 10 & 10 & 5\end{array}\right]$} & $\begin{array}{l}{\left[\begin{array}{llllll}10 & 10 & 10 & 10 & 3 & 3\end{array}\right]} \\
{\left[\begin{array}{llllll}10 & 10 & 10 & 10 & 3 & 2\end{array}\right]}\end{array}$ & {$\left[\begin{array}{llllll}10 & 10 & 10 & 5 & 5 & 5\end{array}\right]$} & $\begin{array}{l}{\left[\begin{array}{lllllll}10 & 10 & 10 & 5 & 5 & 3 & 3\end{array}\right]} \\
{\left[\begin{array}{llllll}10 & 10 & 10 & 5 & 5 & 3\end{array}\right]}\end{array}$ \\
\hline$[10]$ & $\lambda(3)$ & - & - & $\frac{1-3 \lambda}{27}(27)$ \\
\hline$[5]$ & - & - & $\sigma(4)$ & $\frac{1-4 \sigma}{24}(24)$ \\
\hline$[3 / 2]$ & - & - & - & $\frac{1}{24}(24)$ \\
\hline
\end{tabular}

The following equations must hold

$$
\begin{aligned}
x & =\frac{1}{10}(1-3 x-y)+\frac{3}{10}\left(3 \lambda+\frac{2}{3}(1-3 \lambda)\right) x+\frac{6}{10} \frac{3}{4} x \\
y & =\frac{1}{10}(1-4 x)+\frac{4}{10}\left(\lambda+\frac{2}{3}(1-3 \lambda)\right) y+\frac{2}{10}\left(4 \sigma+\frac{1}{2}(1-4 \sigma)\right) y+\frac{3}{10} \frac{2}{3} y \\
z & =\frac{1}{10}(1-4 x-z)+\frac{4}{10} \frac{2}{3}(1-3 \lambda) z+\frac{3}{10} \frac{2}{3}(1-4 \sigma) z+\frac{2}{10} \frac{1}{2} z \\
x & =2 y=4 z
\end{aligned}
$$

${ }^{4}$ The number of minimal winning coalitions can be checked using the Powerslave
oftware (Pajala, 2002). 
The solution to this system is $\lambda=\frac{1}{4}, \sigma=\frac{5}{24}, x=\frac{4}{25}, y=\frac{2}{25}, z=\frac{1}{25}$.

The values of $x$ and $y$ all increase slightly compared with the 1973 values.

This means that if enlargement were put to the vote under weighted majority it would be approved!

Expected equilibrium payoffs are summarized in the following table

$\begin{array}{llll} & \mathbf{1 9 5 8} & \mathbf{1 9 7 3} & \mathbf{1 9 8 1} \\ \text { Germany } & 0.238 & 0.159 & \mathbf{0 . 1 6 0} \\ \text { Italy } & 0.238 & 0.159 & \mathbf{0 . 1 6 0} \\ \text { France } & 0.238 & 0.159 & \mathbf{0 . 1 6 0} \\ \text { Netherlands } & 0.119 & 0.079 & \mathbf{0 . 0 8 0} \\ \text { Belgium } & 0.119 & 0.079 & \mathbf{0 . 0 8 0} \\ \text { Luxemburg } & 0.048 & \mathbf{0 . 0 6 3} & 0.040 \\ \text { UK } & - & 0.159 & \mathbf{0 . 1 6 0} \\ \text { Denmark } & - & 0.071 & 0.040 \\ \text { Ireland } & - & 0.071 & 0.040 \\ \text { Greece } & - & - & 0.080\end{array}$

Table 2. Expected equilibrium payoffs

For comparison, the Shapley value and the Banzhaf index ${ }^{5}$ are

Shapley value

Banzhaf index

$\begin{array}{lllllll}\text { Country } & \mathbf{1 9 5 8} & \mathbf{1 9 7 3} & \mathbf{1 9 8 1} & \mathbf{1 9 5 8} & \mathbf{1 9 7 3} & \mathbf{1 9 8 1} \\ \text { Germany } & 0.233 & 0.179 & 0.174 & 0.238 & 0.167 & 0.158 \\ \text { Italy } & 0.233 & 0.179 & 0.174 & 0.238 & 0.167 & 0.158 \\ \text { France } & 0.233 & 0.179 & 0.174 & 0.238 & 0.167 & 0.158 \\ \text { Netherlands } & 0.150 & 0.081 & 0.071 & 0.143 & 0.091 & 0.082 \\ \text { Belgium } & 0.150 & 0.081 & 0.071 & 0.143 & 0.091 & 0.082 \\ \text { Luxemburg } & 0 & \mathbf{0 . 0 0 1} & \mathbf{0 . 0 3 0} & 0 & \mathbf{0 . 0 1 6} & \mathbf{0 . 0 4 1} \\ \text { UK } & - & 0.179 & 0.174 & - & 0.167 & 0.158 \\ \text { Denmark } & - & 0.057 & 0.030 & - & 0.066 & 0.041 \\ \text { Ireland } & - & 0.057 & 0.030 & - & 0.066 & 0.041 \\ \text { Greece } & - & - & 0.071 & - & - & 0.082\end{array}$

${ }^{5}$ The table reports the normalized Banzhaf index; the effects of enlargement according to the absolute Banzhaf index are qualitatively similar. 
Power indices like the Shapley value and the Banzhaf index agree with the noncooperative model in that the 1973 enlargement favored Luxemburg, and the 1981 enlargement hurt Denmark and Ireland the most. An important difference is that Luxemburg gains in both enlargements according to the power indices, and loses in the second enlargement according to the noncooperative bargaining model. Also, if countries wanted to maximize their Shapley or Banzhaf power indices and enlargement was subject to weighted majority voting, it would have been rejected.

\section{Concluding remarks}

The paradox of new members in the EU is not an artifact of power indices, but can occur in a noncooperative model of bargaining over a fixed pie. In fact, it is stronger in the noncooperative model since enlargement can benefit a majority of existing members.

It is difficult to know the real effects of enlargement. Power indices and the legislative bargaining model agree that the paradox is possible, but differ on which country benefits. The Banzhaf index assumes yes/no voting over exogenous proposals with each country being equally likely to vote yes or no and countries voting independently. The Shapley value may be interpreted as a measure of expected payoffs in bargaining over a fixed pie, though it is difficult to find a compelling bargaining model that yields the Shapley value for weighted majority games. ${ }^{6}$ It is clear that neither yes/no voting with a random agenda and random preferences nor pure bargaining over a private good are accurate models of voting in the Council of Ministers. However, the fact that very different assumptions all lead to the paradox of new members seem to indicate that this is a potentially important phenomenon. The paradox has also been observed experimentally under two different bargaining procedures by Montero, Sefton and Zhang (2008) and Drouvelis, Montero and Sefton (2007).

\footnotetext{
${ }^{6}$ Existing models either assume that all proposals must be passed by unanimity or their results are restricted to a domain that does not include weighted majority games; see the discussion in Drouvelis, Montero and Sefton (2007).
} 


\section{References}

[1] Banks, J.S., and J. Duggan (2000). A Bargaining Model of Collective Choice. American Political Science Review 94:73-88.

[2] Baron, D.P., and J.A. Ferejohn. (1989). Bargaining in Legislatures. American Political Science Review 83:1181-1206.

[3] Brams, S.J., and P.J. Affuso. (1976). Power and Size: a New Paradox. Theory and Decision 7:29-56.

[4] Brams, S.J., and P.J. Affuso. (1985a). New Paradoxes of Voting Power on the EC Council of Ministers. Electoral Studies 4:135-39.

[5] Brams, S.J., and P.J. Affuso. (1985b) Addendum to: New Paradoxes of Voting Power on the EC Council of Ministers. Electoral Studies 4:290.

[6] Drouvelis, M., Montero, M. and Sefton, M. (2007).The Paradox of New Members: Strategic Foundations and Experimental Evidence. CeDEx Discussion Paper 2007-13.

[7] Eraslan, H. and McLennan, A. (2006) Uniqueness of Stationary Equilibrium Payoffs in Coalitional Bargaining. Typescript.

[8] Felsenthal, D.S., and Machover, M., (1998). The Measurement of Voting Power: Theory and Practice, Problems and Paradoxes, Edward Elgar Publishing.

[9] Felsenthal, D. S. and Machover, M. (2001). The Treaty of Nice and Qualified Majority Voting. Social Choice and Welfare 18:431-464.

[10] Montero, M., Sefton, M. and Zhang, P. (2008). Enlargement and the Balance of Power: An Experimental Study. Social Choice and Welfare 30:69-87.

[11] Pajala A. (2002) Power Index Website: a Voting Power WWWResource Including POWERSLAVE Power Index Calculator. [online]. Published 22.4.2002. University of Turku. Turku. <URL:http://powerslave.val.utu.fi/>. 